\title{
Modeling a Quantum Hall System via Elliptic Equations
}

\author{
Artur Sowa \\ Department of Mathematics and Statistics, University of Saskatchewan, 106 Wiggins Road, \\ Saskatoon, SK, Canada S7N 5E6 \\ Correspondence should be addressed to Artur Sowa, sowa@math.usask.ca
}

Received 19 June 2008; Revised 22 August 2008; Accepted 8 September 2008

Recommended by Shao-Ming Fei

Quantum Hall systems are a suitable theme for a case study in the general area of nanotechnology. In particular, it is a good framework to search for universal principles relevant to nanosystem modeling and nanosystem-specific signal processing. Recently, we have been able to construct a partial differential equations-based model of a quantum Hall system, which consists of the Schrödinger equation supplemented with a special-type nonlinear feedback loop. This result stems from a novel theoretical approach, which in particular brings to the fore the notion of quantum information. Here we undertake to modify the original model by substituting the dynamics based on the Dirac operator. This leads to a model that consists of a system of three nonlinearly coupled first-order elliptic equations in the plane.

Copyright (C) 2009 Artur Sowa. This is an open access article distributed under the Creative Commons Attribution License, which permits unrestricted use, distribution, and reproduction in any medium, provided the original work is properly cited.

\section{Quantum Entanglement of Composite Systems and Its Associated Hamiltonian Dynamics}

In this section, we give a very brief overview of the principles behind the mesoscopic loop models as developed in [1-5]. The theme of quantum information is extremely relevant to our approach, but it has been brought to light in explicit terms only recently; an in-depth discussion will appear elsewhere [6, 7].

Since the measurement of Hall resistance in a quantum Hall system results in a classical signal, it is natural to ask if any quantum information is in the process transduced to the classical medium. Let us develop this point of view into a formal discussion. Suppose that the electronic solid-state system and the ambient magnetic field are represented by two Hilbert spaces; say $\mathbf{H}$ and $\widehat{\mathbf{H}}$, respectively. According to the principles of quantum mechanics [8], the combined system is then represented by the tensor product space $\widehat{\mathbf{H}} \otimes \mathbf{H}$. Moreover, the states of the composite system assume the form 


$$
\left|\Psi_{\text {combined }}\right\rangle=\sum_{m, n} A_{m n}^{*}\left|\varphi_{m}\right\rangle \otimes\left|\psi_{n}\right\rangle \in \widehat{\mathbf{H}} \otimes \mathbf{H}
$$

We will associate with every state as above an entanglement operator (with no intention to imply that the composite state (1.1) is necessarily entangled in the rigorous sense of the term [8]):

$$
K=\sum A_{m n}\left|\varphi_{m}\right\rangle\left\langle\psi_{n}\right|
$$

Thus, if an electronic system is found in the state $\psi \in \mathbf{H}$, then the electromagnetic system will collapse into the state

$$
K \psi \in \widehat{\mathbf{H}} \text {. }
$$

Let us define the density operator of system $\mathbf{H}$ in the usual way:

$$
\rho=\operatorname{Tr}_{\hat{\mathbf{H}}}\left(\left|\Psi_{\text {combined }}\right\rangle\left\langle\Psi_{\text {combined }}\right|\right)
$$

One readily finds

$$
\rho=K^{*} K
$$

This lays out the vocabulary for a quantum-mechanical description of a composite system. Next, we postulate a model relevant specifically to the quantum Hall systems. Namely, we propose that, in the presence of a magnetic field, the dynamics of the electronic system are derived from the energy functional of the form

$$
\operatorname{Tr}(H \rho)+\beta \log \operatorname{det}(\rho)
$$

where $H$ is the "regular" Hamiltonian governing the microscopic dynamics of the electronic subsystem. For the purposes at hand, there is no need to discuss the constant $\beta$ beyond the simple statement that it is nonzero when the magnetic field is nonvanishing. We would like to point out that this energy functional is a special case of functionals given in the form, say, $\operatorname{Tr}(H \rho)+\beta \operatorname{Tr}(f(\rho))$, where $f$ is a suitable analytic function. Indeed, log $\operatorname{det}(\rho)=$ $\operatorname{Tr}(\log \rho)$. Roughly speaking, function $f$ describes the interaction between two subsystems of a composite quantum system. Many different aspects of the general models of this kind are discussed in [6,7]. As it is shown in [4], and again here, the functional with $f=\log$ captures the characteristic of a quantum Hall system, which justifies our special interest in it. It is also worthwhile mentioning that $-\operatorname{Tr}(\log \rho)$ is the von Neumann relative quantum entropy $S(I|| \rho)[8]$.

Henceforth we assume for simplicity that $H$ is diagonalizable in the basis consisting of its eigenvalues:

$$
H \psi_{k}=E_{k} \psi_{k} .
$$


Now, substituting the decomposition (1.5) into (1.6) yields the following Hamiltonian:

$$
\Xi(K)=\operatorname{Tr}\left(K H K^{*}\right)+\beta \log \operatorname{det}\left(K^{*} K\right) .
$$

This in turn leads to the following system of Hamiltonian equations (see [1, 2]):

$$
-i \hbar \dot{K}=K H+\beta K^{*-1} \text {. }
$$

(From this point on we will set $\hbar=1$.) The corresponding eigenvalue problem assumes the form

$$
K H+\beta K^{*-1}=v K .
$$

Equation (1.11) is a part of the proposed model of quantum Hall systems in homeostasis. Solutions of (1.10) are found to assume the form (see [1,2])

$$
K=\sum_{E_{k}<\nu}\left(\frac{\beta}{v-E_{k}}\right)^{1 / 2}\left|U \psi_{k}\right\rangle\left\langle\psi_{k}\right|,
$$

where $U: \mathbf{H} \rightarrow \widehat{\mathbf{H}}$ is an arbitrary unitary transformation.

\section{The Mesoscopic-Loop Model Based on the Dirac-Type Equations}

The model we are about to present deals with two types of particles, which will be called electrons and holes. Both of them will have the same effective mass $m^{*}=1$ and charge $e=1$. It seems appropriate to emphasize that, of course, the notion of effective mass depends on the model. All analyses will be performed in a two-dimensional plane. Recall that the Hodge star $*$ in two dimensions is a linear operation on differential forms determined by the relations $* d x=d y, * d y=-d x, * 1=d x \wedge d y$, and $*(d x \wedge d y)=1$. The exterior derivative $d$ defines the coderivative $\delta=-* d *$. Let the vector potential $A$ be given in local coordinates as $A=$ $A_{1} d x+A_{2} d y$. In particular, $\delta A=-\left(A_{1, x}+A_{2, y}\right)$, while

$$
B(x, y)=* d A=A_{2, x}-A_{1, y}
$$

is the magnetic flux density. The corresponding Schrödinger operator $S_{A}$ acts on a wave function $\psi$ as follows:

$$
2 S_{A} \psi=-\left(\partial_{x}^{2}+\partial_{y}^{2}\right) \psi+2 i\left(A_{1} \partial_{x} \psi+A_{2} \partial_{y} \psi\right)+\left(A_{1}^{2}+A_{2}^{2}\right) \psi-i(\delta A) \psi
$$

(The factor of 2 in front of $S_{A}$ stems from the fact that in the adopted units the kinetic energy is multiplied by a factor of $\hbar^{2} / 2 m^{*}=1 / 2$.) The Schrödinger operator can be successfully used in a construction of mesoscopic loop models [5]. In this paper, we will consider a modification 
based on a Dirac-type factorization of the Schrödinger operator. To this end, we use the twodimensional Dirac operator [9] with its parameters set at $m=0$ and $c=1$. Namely, let

$$
H_{A}=\left[\begin{array}{cc}
0 & D^{*} \\
D & 0
\end{array}\right]
$$

where

$$
D=-i \partial_{x}-A_{1}+\partial_{y}-i A_{2}
$$

so that

$$
D^{*}=-i \partial_{x}-A_{1}-\partial_{y}+i A_{2}
$$

A direct calculation shows that

$$
H_{A}^{2}=\left[\begin{array}{cc}
D^{*} D & 0 \\
0 & D D^{*}
\end{array}\right]=\left[\begin{array}{cc}
2 S_{A}-B & 0 \\
0 & 2 S_{A}+B
\end{array}\right],
$$

where $B=B(x, y)$ is determined by $A$ via (2.1). Observe the difference of signs at the magnetic flux density term. This necessitates the interpretation of the two wave functions defining the state vector

$$
\left|\begin{array}{l}
\psi_{1} \\
\psi_{2}
\end{array}\right\rangle
$$

as representing particles with opposite pseudospins. At this point, we venture to describe a quantum Hall system in a manner discussed in the previous section, which brings the notion of entanglement to the fore. Namely, we consider the following system of equations:

$$
\begin{gathered}
H_{A}\left|\begin{array}{l}
\psi_{1} \\
\psi_{2}
\end{array}\right\rangle=E\left|\begin{array}{l}
\psi_{1} \\
\psi_{2}
\end{array}\right\rangle, \\
\left.* d A(x, y)=|K| \begin{array}{l}
\psi_{1} \\
\psi_{2}
\end{array}\right\rangle\left.(x, y)\right|^{2},
\end{gathered}
$$

which involves a coupling realized via the entanglement transform $K$. Note that this forces an appropriate normalization of $K$ so as to guarantee the correct charge to flux ratio. 


\section{Quantization of Hall Resistance}

Note that if $\left.\left.\right|_{\psi_{2}} ^{\psi_{1}}\right\rangle$ is a solution of (2.8), then in particular it is an eigenfunction of $H_{A}$. We now request that $K$ be a stationary solution as in (1.11) with $U=I$, where

$$
\left|\psi_{k}\right\rangle=\left|\begin{array}{l}
\psi_{k, 1} \\
\psi_{k, 2}
\end{array}\right\rangle
$$

are the eigenvectors of the Dirac Hamiltonian $H_{A}$. In general, properties of the model depend on the choice of $U$. The case $U=I$ is interpreted as corresponding to the phasecorrelated regime; see [1] for a more detailed discussion of this point. Next, assume $v>E$. Without loss of generality, we may also assume that $\left|\psi_{\psi_{2}}^{\psi_{1}}\right\rangle$ is on the list of mutually orthogonal eigenfunctions defining $K$ via (1.11). (This can always be arranged even if $E$ has multiplicity greater than 1.) Thus, we have

$$
K\left|\begin{array}{l}
\psi_{1} \\
\psi_{2}
\end{array}\right\rangle=c\left|\begin{array}{l}
\psi_{1} \\
\psi_{2}
\end{array}\right\rangle
$$

for a constant $c$. Consequently,

$$
\left.|K| \begin{array}{l}
\psi_{1} \\
\psi_{2}
\end{array}\right\rangle\left.(x, y)\right|^{2} \sim\left|\psi_{1}\right|^{2}+\left|\psi_{2}\right|^{2}
$$

This implies that the last equation of (2.8) is equivalent to the statement

$$
\partial_{x} A_{2}-\partial_{y} A_{1}=R_{H}\left(\left|\psi_{1}\right|^{2}+\left|\psi_{2}\right|^{2}\right) .
$$

Note that $R_{H}$ is the ratio of the total flux to the total charge counted between the two types of particles. In particular, due to quantization of flux and charge, $R_{H}$ is a rational number when expressed in appropriate units. We will demonstrate that in a symmetric realization of this model the Hall resistance is equal to $2 R_{H}$. We conduct the analysis in a rectangular-domain setting with the assumption that the wave functions are periodic in $x$, both with the same period; that is,

$$
\begin{aligned}
& \psi_{1}=\exp (i k x) \chi_{1}(y), \\
& \psi_{2}=\exp (i k x) \chi_{2}(y) .
\end{aligned}
$$

By substituting to (2.8), we readily obtain

$$
\begin{gathered}
X_{1}^{\prime}=-\left(k-A_{1}\right) \chi_{1}+E_{X_{2}}, \\
X_{2}^{\prime}=\left(k-A_{1}\right) \chi_{2}-E_{X_{1}} .
\end{gathered}
$$


Furthermore, let us assume $A_{2}=0, A_{1}=A_{1}(y)$, and $A_{1}(0)=0$. Let us also introduce an auxiliary variable $w=k-A_{1}$. In such a case, (2.8) is equivalent to the system

$$
\begin{gathered}
w^{\prime}=R_{H}\left(\chi_{1}^{2}+\chi_{2}^{2}\right), \quad w(0)=k, \\
X_{1}^{\prime}=-w_{\chi_{1}}+E_{X_{2},} \\
X_{2}^{\prime}=w_{X_{2}}-E_{X_{1}} .
\end{gathered}
$$

By differentiation, we readily obtain

$$
\begin{aligned}
& \chi_{1}^{\prime \prime}=\left(-E^{2}+w^{2}-w^{\prime}\right) \chi_{1}, \\
& \chi_{2}^{\prime \prime}=\left(-E^{2}+w^{2}+w^{\prime}\right) \chi_{2} .
\end{aligned}
$$

These are 1D stationary Schrödinger equations with energy $E^{2} / 2$. Note that $\chi_{1}$ is affected by potential $\left(w^{2}-w^{\prime}\right) / 2$, while $\chi_{2}$ is affected by potential $\left(w^{2}+w^{\prime}\right) / 2$. Both quantities result from the presence of the magnetic field, and thus they represent the Hall effect. There are two potential functions, differing by the sign of the correction term $w^{\prime}$, because two types of carriers are involved in the charge transport. Note that

$$
w(y)=k+R_{H} \int_{0}^{y}\left(x_{1}^{2}\left(y^{\prime}\right)+x_{2}^{2}\left(y^{\prime}\right)\right) d y^{\prime}
$$

We calculate the current next. Recall that

$$
j=\operatorname{Re}\left\{\psi_{1}^{*}\left(i \cdot d \psi_{1}+\psi_{1} A\right)\right\}+\operatorname{Re}\left\{\psi_{2}^{*}\left(i \cdot d \psi_{2}+\psi_{2} A\right)\right\} .
$$

A direct calculation shows that the two 1 -forms amount to

$$
\operatorname{Re}\left\{\psi_{l}^{*}\left(i \cdot d \psi_{l}+\psi_{l} A\right)\right\}=-x_{l}^{2}(y) w(y) d x, \quad l=1,2 .
$$

In summary,

$$
j=-\frac{1}{R_{H}} w^{\prime}(y) w(y) d x=\frac{1}{R_{H}} * d \frac{w^{2}}{2} .
$$

(We remind the reader that $*$ denotes the Hodge star.) Observe that the current flows along the $x$-axis. Since both types of carriers contribute to the current, the total Hall potential is the sum of the two separate potentials pointed out above, that is,

$$
V_{H}=\frac{w^{2}+w^{\prime}}{2}+\frac{w^{2}-w^{\prime}}{2}=w^{2}
$$


It follows that

$$
j=\frac{1}{2 R_{H}} * d V_{H}
$$

By integration, we then obtain that the Hall resistance is $2 R_{H}$. We remark that this is twice the value obtained in the Schrödinger-type model (cf. $[4,5]$ ). Recall that $R_{H}$ has been introduced as the ratio of the number of quanta of the magnetic field to the number of quanta of electric charge $e=1$ of both types of particles. If particles of type, say, $\psi_{2}$ were not accounted for in the calculation of the filling factor, then there would be a discrepancy between the inverse filling factor and the quantity $R_{H}$.

It is interesting to ask when the electrons and holes are engaged as charge carriers in equal numbers. Let us position the edges of the plate symmetrically about the $x$-axis, that is, $y \in[-b, b]$. Note that the number of carriers of each type is proportional to

$$
\int_{-b}^{b} x_{l}^{2}\left(y^{\prime}\right) d y^{\prime}, \quad l=1,2
$$

Now assume $\chi_{2}(0)=\chi_{1}(0)$, and consider the last two equations of (3.7). In such a case, one can find a solution satisfying the condition $x_{2}(y)=x_{1}(-y)$. For this solution, particles of each type occur in equal numbers.

\section{The Elliptic System in the Plane}

In Section 3, we demonstrated that Hall resistance is quantized under certain symmetry assumptions which reduce the problem to a system of ordinary differential equations. We emphasize that Hall potentials and Hall resistance can be defined and discussed in the general two-dimensional setting [5]. In fact, the two-dimensional, that is, partial differential equations (PDEs), setting cannot be avoided if we want to study the effect of lattice potential and so forth. We will now briefly discuss the ramifications of the PDE problem. Consider (2.8) jointly with the assumptions about $K$ put forth in the previous section. In this case, it is equivalent to the following system of first-order partial differential equations:

$$
\begin{gathered}
\partial_{y} \psi_{1}=i \partial_{x} \psi_{1}+\left(A_{1}+i A_{2}\right) \psi_{1}+E \psi_{2}, \\
\partial_{y} \psi_{2}=-i \partial_{x} \psi_{2}-\left(A_{1}-i A_{2}\right) \psi_{2}-E \psi_{1}, \\
\partial_{y} A_{1}=\partial_{x} A_{2}-R_{H}\left(\left|\psi_{1}\right|^{2}+\left|\psi_{2}\right|^{2}\right) .
\end{gathered}
$$

A direct calculation shows that if a triplet $\left(\psi_{1}, \psi_{2}, A\right)$ is a solution of the system, then so is $\left(e^{i \varphi} \psi_{1}, e^{i \varphi} \psi_{2}, A+d \varphi\right)$ for an arbitrary real function $\varphi=\varphi(x, y)$. Function $\varphi$ represents gauge freedom and therefore carries no physical information. In particular, only the relative phase of the pair $\left(\psi_{1}, \psi_{2}\right)$ is physically meaningful. Next, observe that while system (4.1) is not elliptic by itself, it will become strictly elliptic when amended with the gauge-fixing equation

$$
\partial_{y} A_{2}=-\partial_{x} A_{1}
$$




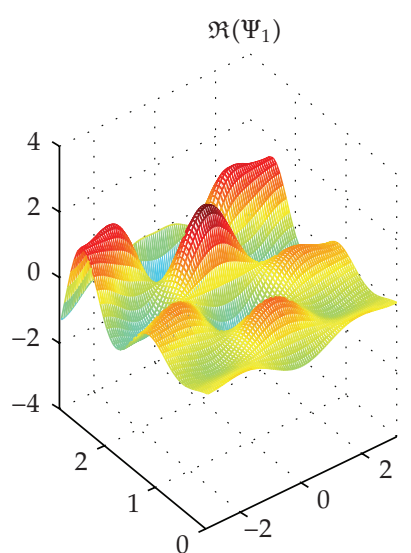

(a)

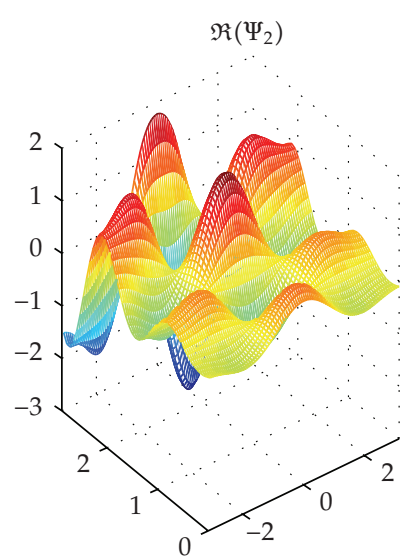

(b)

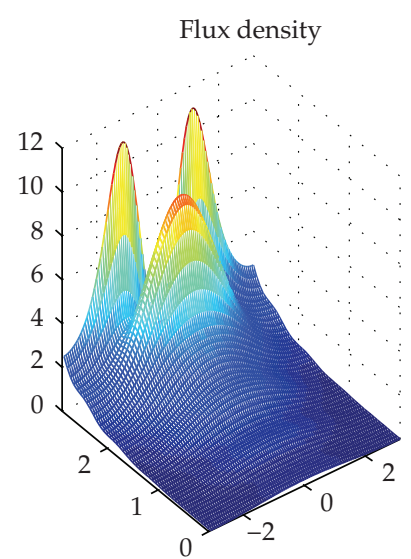

(c)

Figure 1: A numerical solution of (4.1) with $E=5$ (in units adopted throughout the paper), $R_{H}=3 / 5$, and $A_{2} \equiv 0$. The initial values $\psi_{2}(x, 0)=0.3 \exp (i x)+0.2 * \exp (2 i x), \psi_{1}(x, 0)=-0.9 \psi_{2}(x, 0)$, and $A_{1}(x, 0)=0$ are prescribed on the edge facing front right.

Consider a system that consists of (4.1) and (4.2). Note that the nonlinear terms in (4.1) are (real) analytic functions of the dependent variables. Therefore, the classical CauchyKovalevskaya theorem [10] guarantees local existence of solutions of the initial value problem when the boundary conditions are also analytic. The initial value problem may help gain some insight into the nature of the system; an example of a solution obtained numerically with a finite difference method is displayed in Figure 1. Since the boundary value problems spring to mind in this context, one should emphasize that in view of ellipticity, the natural setting is that of the Riemann-Hilbert-type problem [11]. However, we wish to signal briefly that the boundary value problem is not the only type of approach appropriate for the study of the mesoscopic-loop model; there is a promising complementary approach. Namely, setting $\mathrm{A}=A_{1}+i A_{2}$, we can represent (4.1)-(4.2) as a system of three nonlinearly coupled $\bar{\partial}$-equations:

$$
\begin{gathered}
\bar{\partial} \psi_{1}=\frac{i}{2}\left(\mathrm{~A} \psi_{1}+E \psi_{2}\right), \\
\bar{\partial} \bar{\psi}_{2}=-\frac{i}{2}\left(\mathrm{~A} \bar{\psi}_{2}+E \bar{\psi}_{1}\right), \\
\bar{\partial} \overline{\mathrm{A}}=-\frac{i}{2} R_{H}\left(\left|\psi_{1}\right|^{2}+\left|\psi_{2}\right|^{2}\right) .
\end{gathered}
$$

This suggests the relevance of a variety of complex methods [12], but this theme will not be discussed in the present paper.

\section{Closing Comments}

The Dirac equation is undergoing a renaissance in condensed matter physics due to its relevance to the unusual two-dimensional crystal called graphene (see, e.g., [13]). I emphasize that in this paper we have not addressed any of the issues related to that stream of 
research. Rather, we have used the Dirac equation as an analytic alternative to the Schrödiner equation. It helped us find an almost equivalent reformulation of the nonrelativistic model.

\section{Acknowledgment}

The author is grateful to the journal referees whose thoughtful questions and comments helped improve this paper.

\section{References}

[1] A. Sowa, "Mesoscopic mechanics," Journal of Physics and Chemistry of Solids, vol. 65, no. 8-9, pp. 15071515, 2004.

[2] A. Sowa, "Integrability in the mesoscopic dynamics," Journal of Geometry and Physics, vol. 55, no. 1, pp. 1-18, 2005.

[3] A. Sowa, "Parametric models of the Hall effect in the strongly correlated regime," Waves in Random and Complex Media. In press.

[4] A. Sowa, "Fractional quantization of Hall resistance as a consequence of mesoscopic feedback," Russian Journal of Mathematical Physics, vol. 15, no. 1, pp. 122-127, 2008.

[5] A. Sowa, "Sensitivity to lattice structure in the mesoscopic-loop models of planar systems," Advanced Studies in Theoretical Physics, vol. 1, no. 9-12, pp. 433-448, 2007.

[6] A. Sowa, "Quantum entanglement in composite nanosystems," submitted.

[7] A. Sowa, "Nonlocal bonding in composite quantum systems," submitted.

[8] S. Stenholm and K.-A. Suominen, Quantum Approach to Informatics, John Wiley \& Sons, New York, NY, USA, 2005.

[9] B. Thaller, The Dirac Equation, Texts and Monographs in Physics, Springer, Berlin, Germany, 1992.

[10] R. Courant and D. Hilbert, Methods of Mathematical Physics. Vol. II, Wiley Classics Library, John Wiley \& Sons, New York, NY, USA, 1989.

[11] W. L. Wendland, Elliptic Systems in the Plane, vol. 3 of Monographs and Studies in Mathematics, Pitman, Boston, Mass, USA, 1979.

[12] S. G. Krantz, Geometric Function Theory: Explorations in Complex Analysis, Cornerstones, Birkhäuser, Boston, Mass, USA, 2006.

[13] K. S. Novoselov, A. K. Geim, S. V. Morozov, et al., “Two-dimensional gas of massless Dirac fermions in graphene," Nature, vol. 438, no. 7065, pp. 197-200, 2005. 


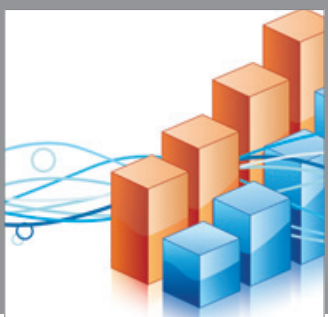

Advances in

Operations Research

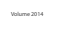

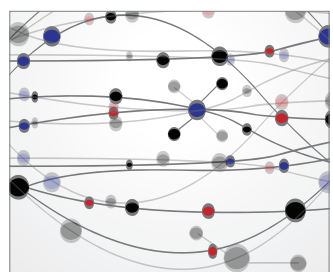

\section{The Scientific} World Journal
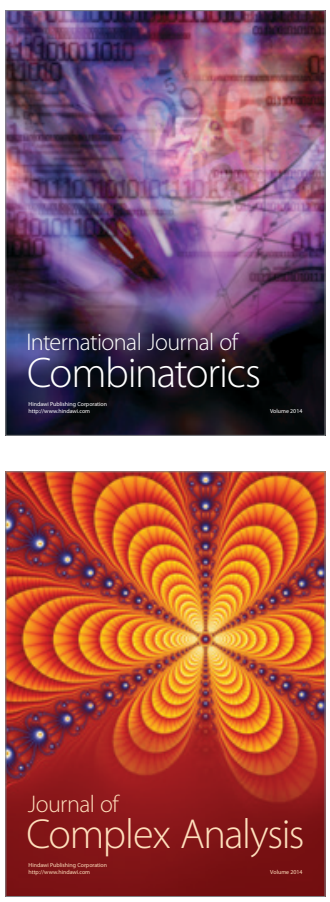

International Journal of

Mathematics and

Mathematical

Sciences
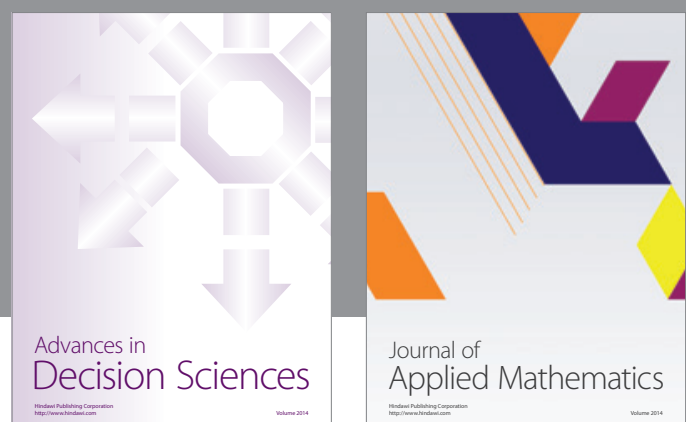

Journal of

Applied Mathematics
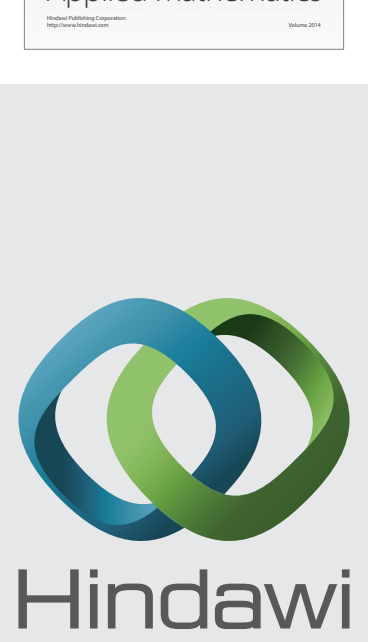

Submit your manuscripts at http://www.hindawi.com
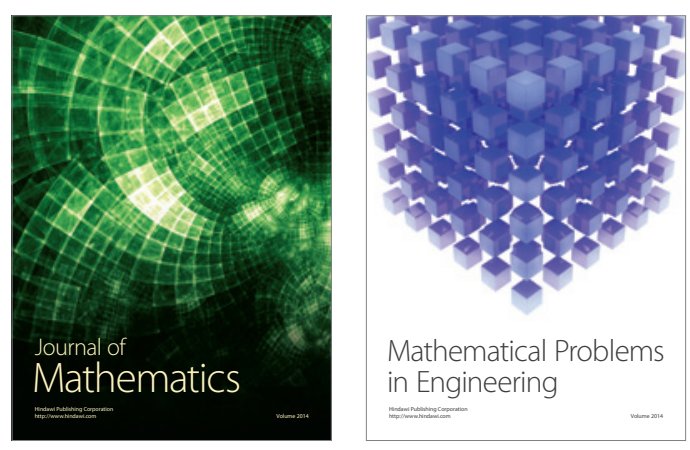

Mathematical Problems in Engineering
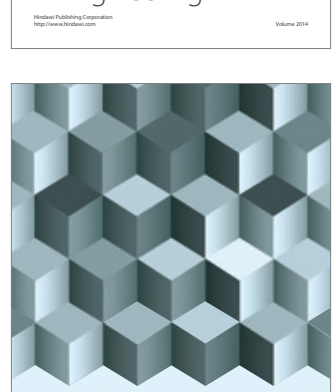

Journal of

Function Spaces
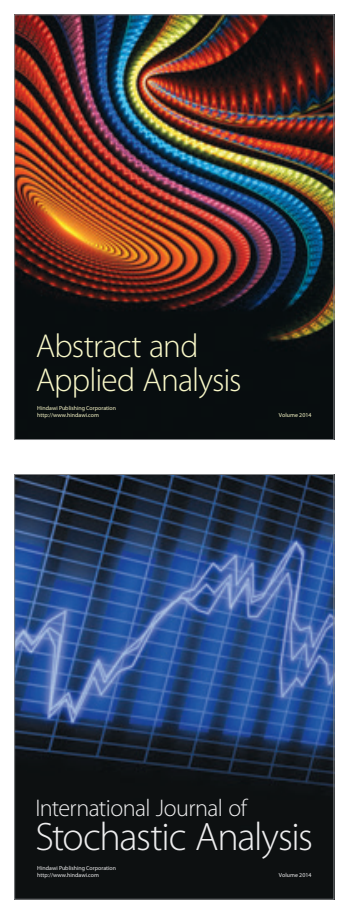

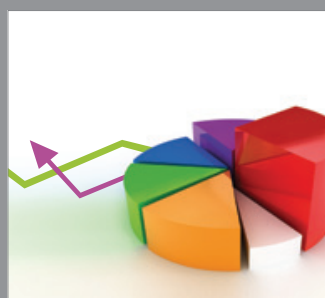

ournal of

Probability and Statistics

Promensencen
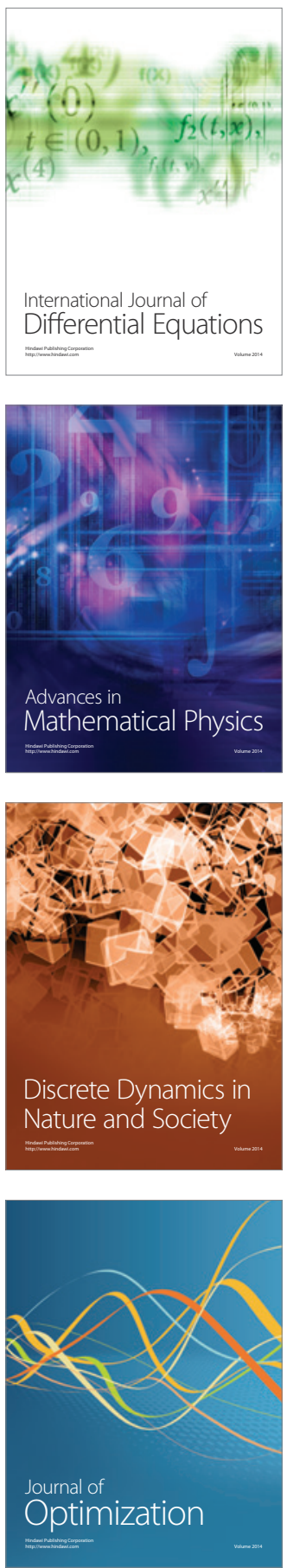